\title{
126. On Equivalence of Modular Function Spaces
}

\author{
By Jyun IsHII \\ Mathematical Department, Hokkaidô University, Sapporo \\ (Comm. by K. KunUgi, M.J.A., Nov. 12, 1959)
}

Let $\Omega$ be an abstract space and $\mu$ be a totally additive measure defined on a totally additive set class $\mathfrak{B}$ of subsets of $\Omega$ satisfying $\bigcup_{\mu(B)<\infty} E=\Omega$.

Let $\Phi(\xi, \omega)(\xi \geqq 0, \omega \in \Omega)$ be a function satisfying the following conditions:

1) $0 \leqq \Phi(\xi, \omega) \leqq \infty \quad$ for all $\xi \geqq 0, \omega \in \Omega$;

2) $\Phi(\xi, \omega)$ is a measurable function on $\Omega$ for all $\xi \geqq 0$;

3) $\Phi(\xi, \omega)$ is a non-decreasing convex functions of $\xi \geqq 0$ for all $\omega \in \Omega$

4) $\Phi(0, \omega)=0$ for all $\omega \in \Omega$;

5) $\Phi(\alpha-0, \omega)=\Phi(\alpha, \omega)$ for all $\omega \in \Omega$;

6) $\Phi(\xi, \omega) \rightarrow \infty$ as $\xi \rightarrow \infty$ for all $\omega \in \Omega$;

7) for any $\omega \in \Omega$, there exists $\alpha_{\omega}>0$ such that $\Phi\left(\alpha_{\omega}, \omega\right)<\infty$.

For any measurable function $x(\omega) \quad(\omega \in \Omega), \quad \Phi(|x(\omega)|, \omega)$ is also measurable. We shall denote by $L_{\phi}(\Omega)$ the class of all measurable functions $x(\omega)(\omega \in \Omega)$ such that, for some $\alpha=\alpha_{x}>0$,

$$
\int_{\Omega} \Phi(\alpha|x(\omega)|, \omega) d \mu(\omega)<\infty .{ }^{1)}
$$

We write $x \geqq y\left(x, y \in L_{\oplus}\right)$, if $x(\omega) \geqq y(\omega)$ for a.e. ${ }^{2)}$ on $\Omega$, then $L_{\emptyset}$ is a universally continuous semi-ordered linear space.

If we define a functional

$$
m_{\Phi}(x)=\int_{\Omega} \Phi(|x(\omega)|, \omega) d \mu,
$$

$m_{\varnothing}$ satisfies all the modular conditions and furthermore $m_{\emptyset}$ is monotone complete. Such a space $L_{\Phi}$ with $m_{\Phi}$ is said to be a modular function space. $^{3)}$

If $\bar{\Phi}(\eta, \omega)(\eta \geqq 0, \omega \in \Omega)$ is, for every fixed $\omega \in \Omega$, the complementary function of $\Phi$ in the sense of $\mathrm{H}$. W. Young, $\bar{\Phi}$ satisfies all the corresponding properties from 1) to 7) on $\Phi$, and so, we have also a

1) For the integration, refer, for instance, H. Nakano [4].

2) Here "a.e. (almost everywhere)" means always that "except on some $A \in \mathfrak{B}$ which $\mu(E \cap A)=0$ for all $\mu(E)<\infty$ ".

3) Modulared function spaces were defined and discussed in H. Nakano [2, Appendices I, II]. For all other definitions and notations used in this note, see the same book, too. 
modular function space $L_{\bar{\Phi}}$ with $m_{\bar{\Phi}}$, which is isometric to the conjugate modular space of $L_{\Phi}$ with $m_{\Phi}$.

For two functions $\Phi$ and $\Psi$ on $\Omega$ satisfying above the conditions, we say that $L_{\Phi}$ is equivalent to $L_{\Psi}$, if $L_{\Phi}=L_{\Psi}$.

In this note, we find a necessary and sufficient condition in order that a modular function space is equivalent to the other. Immediate consequence of the fact gives the condition in order that a modular function space is equivalent to an Orlicz space.

Lemma.) Let $R$ be an abstract modular space with two modulars $m_{1}$ and $m_{2}$, and $m_{1}$ be monotone complete.

( I ) There exist $\varepsilon, \varepsilon^{\prime}, k, K, \gamma>0$ such that
$m_{2}(k x)<\gamma$
for all $x$ with $m_{1}(x)<\varepsilon$
(b)
$m_{2}(k x) \leqq K m_{1}(x)$
for all $x$ with $\varepsilon^{\prime} \leqq m_{1}(x)<\varepsilon$.

(II) If $R$ is non-atomic, there exist $\varepsilon, k, K>0$ such that

$$
m_{2}(k x) \leqq K m_{1}(x) \quad \text { for all } x \text { with } m_{1}(x) \geqq \varepsilon \text {. }
$$

Proof. ( I ). (a) If (a) is not valid, there exists a sequence $0 \leqq x_{\nu} \in R(\nu=1,2, \cdots)$ such that $m_{1}\left(x_{\nu}\right) \leqq \frac{1}{2^{\nu}}, m_{2}\left(\frac{1}{\nu} x_{\nu}\right) \geqq \nu$. Let $y_{n}=\bigcup_{\nu=1}^{n} x_{\nu}$ $(n=1,2, \cdots)$, then $0 \leqq y_{n} \uparrow_{n=1}^{\infty}$ and $m_{1}\left(y_{n}\right) \leqq 1(n=1,2, \cdots)$. Therefore there exists $y_{0}=\bigcup_{n=1}^{\infty} y_{n}$, because $m_{1}$ is monotone complete. On the other hand, for $\nu=1,2, \cdots \quad m_{2}\left(\frac{1}{\nu} y_{0}\right) \geqq m_{2}\left(\frac{1}{\nu} y_{n}\right) \geqq n \quad$ for $n \geqq \nu$,

which contradicts that $m_{2}$ is a modular. by (a).

(b) For any $x$ with $\frac{\varepsilon}{2} \leqq m_{1}(x)<\varepsilon$, we have $m_{2}(k x) \leqq \gamma \leqq \frac{2 \gamma}{\varepsilon} m_{1}(x)$

(II) Let $\varepsilon$ be the same as in (I). If $m_{1}(x) \geqq \varepsilon$, there exists an integer $n$ such that $\varepsilon n \leqq m_{1}(x)<\varepsilon(n+1)$. We can decompose $x$ into an orthogonal sequence $x_{\nu}(\nu=1,2, \cdots, n+1)$ such that

$$
x=\sum_{\nu=1}^{n+1} \oplus x_{\nu} \quad \text { and } m_{1}\left(x_{\nu}\right)<\varepsilon,
$$

because $R$ is non-atomic. Therefore

$$
m_{2}(k x)=\sum_{\nu=1}^{n+1} m_{2}\left(k x_{\nu}\right)<2 n \gamma \leqq \frac{2 \gamma}{\varepsilon} m_{1}(x)
$$

by (a) in (I).

First, we consider only the case that $\mu$ is non-atomic.

Theorem 1. $^{5)} \quad L_{\emptyset}(\Omega) \subseteq L_{\Psi}(\Omega)$ if and only if there exist $k, K>0$ and $c(\omega) \in L_{1}(\Omega)$ such that

$$
\Psi(k \xi, \omega) \leqq K \Phi(\xi, \omega)+c(\omega)
$$

for all $\xi \geqq 0$ and a.e. on $\Omega$.

4) The proof of this lemma has relations to results of [1] and [6].

5) This theorem is a generalization of Theorem 1a in [5, Chap. II, §1]. 
Proof. It is clear that (*) implies $L_{\Phi} \subseteq L_{\ddot{t}}$. We prove the converse. Let $0 \leqq \alpha_{\nu}(\nu=1,2, \cdots)$ be the system of all the positive rational numbers. For any $E \in \mathfrak{B}$ with $\mu(E)<\infty$ and for $\varepsilon, k, K>0$ in (II), we put, for $\nu=1,2, \cdots$

(\#)

$$
E_{\nu}=\left\{\omega ; \Psi\left(k \alpha_{\nu}, \omega\right)>K \Phi\left(\alpha_{\nu}, \omega\right)\right\}_{\frown} E
$$

and

$$
x_{\nu}(\omega)=\alpha_{\nu} \chi_{E_{\nu}}(\omega)
$$

respectively, where $\chi_{E_{\nu}}$ is the characteristic function of $E_{\nu}$.

We need to consider only on such $\nu$ that $\mu\left(E_{\nu}\right) \neq 0$ in the following.

Since $\Phi\left(\alpha_{\nu}, \omega\right)<\infty$ on $E_{\nu}$ by (\#), we have

$$
E_{\nu, n}=\left\{\omega ; \Phi\left(\alpha_{\nu}, \omega\right)<n\right\}_{\frown} E_{\nu} \uparrow_{n=1}^{\infty} E_{\nu} .
$$

For all $n \geqq n_{0}$ where $n_{0}$ is sufficiently large such that $\mu\left(E_{\nu, n_{0}}\right) \neq 0$, we have $\alpha_{\nu} \chi_{E_{\nu}, n} \in L_{\emptyset}$ and $m_{\mathscr{\varphi}}\left(\alpha_{\nu} \chi_{E_{\nu}, n}\right)<\varepsilon$, if otherwise, the fact

contradicts (II).

$$
\begin{aligned}
& m_{\Psi}\left(k \alpha_{\nu} \chi_{E_{\nu}, n}\right)=\int_{E_{\nu}, n} \psi\left(k \alpha_{\nu}, \omega\right) d \mu \\
& \quad>K \int_{E_{\nu}, n} \Phi\left(\alpha_{\nu}, \omega\right) d \mu=K m_{\Phi}\left(\alpha_{\nu} \chi_{E_{\nu}, n}\right)
\end{aligned}
$$

Therefore, considering $\alpha_{\nu} \chi_{E_{\nu}, n} \uparrow_{n=1}^{\infty}$ and $m_{\mathscr{D}}\left(\alpha_{\nu} \chi_{E_{\nu}, n}\right)<\varepsilon$, we have $x_{\nu}=$ $\bigcup_{n=1}^{\infty} \alpha_{\nu} \chi_{E_{\nu}, n} \in L_{\emptyset}$ and $m\left(x_{\nu}\right)<\varepsilon$ likewise by (\#) and (II). Here, putting $y_{n}=\bigcup_{\nu=1}^{n} x_{\nu}$, we have a sequence of step functions $0 \leqq y_{n} \uparrow_{n=1}^{\infty}$ where $y_{n}=$ $\sum_{\mu=1}^{n} \alpha_{\nu_{\mu}} \chi_{E^{(\mu)}}$ for $\alpha_{\nu_{1}}<\alpha_{\nu_{2}}<\cdots<\alpha_{\nu_{n}}$ with $\nu_{\mu}=\mu(\mu=1,2, \cdots, n)$ and for the system of disjoint sets $E^{(\mu)}=E_{\nu \mu}-\left(\bigcup_{\rho=1}^{\mu-1} E_{\nu \rho}\right) \frown E_{\nu \mu}$.

Since, for all $n=1,2, \cdots$

$$
\begin{aligned}
m_{\Psi}\left(k y_{n}\right) & =\int_{\Omega} \Psi\left(k \sum_{\rho=1}^{n} \alpha_{\nu \rho} \chi_{E^{(\rho)}}(\omega), \omega\right) d \mu \\
& =\sum_{\mu=1}^{n} \int_{E(\rho)} \psi\left(k \alpha_{\nu \rho}, \omega\right) d \mu>\sum_{\rho=1}^{n} \int_{E(\rho)} k \Phi\left(\alpha_{\nu \rho}, \omega\right) d \mu \\
& =K \int_{\Omega} \Phi\left(\sum_{\rho=1}^{n} \alpha_{\nu \mu} \chi_{E(\rho)}(\omega), \omega\right) d \mu=K m_{\Phi}\left(y_{n}\right),
\end{aligned}
$$

we have $m_{\oplus}\left(y_{n}\right)<\varepsilon$ by (a) and (II).

Therefore $y_{E}=\bigcup_{n=1}^{\infty} y_{n} \in L_{\Phi}$ because $m_{\Phi}$ is monotone complete, and furthermore $m_{\mathscr{\varphi}}\left(y_{E}\right) \leqq \varepsilon$. Namely $y_{E} \in L_{\Psi}$ by the hypothesis and $m_{\Psi}\left(k y_{E}\right)<\gamma$ by (a) in (I).

Now, we have, for all $n=1,2, \cdots$

$$
\Psi\left(k \alpha_{n}, \omega\right) \leqq \begin{cases}\Psi\left(k y_{E}(\omega), \omega\right) & \text { for all } \omega \in E_{n} \\ K \Phi\left(\alpha_{n}, \omega\right) & \text { for all } \omega \in E-E_{n} \frown E .\end{cases}
$$

The system $\left\{y_{E}\right\}$ in which every $y_{E}$ is determined depending on $E \in \mathfrak{B}$ 
with $\mu(E)<\infty$ by the above-stated way constitutes a directed system $0 \leqq y_{E} \uparrow_{\mu(E)<\infty}$, because for any two elements $y_{E}, y_{F} \in\left\{y_{E}\right\}$ we have $\mu(E \bigcup F)<\infty$ and $y_{E} \bigcup y_{F}=y_{E \cup F}$. Since $m\left(y_{E}\right) \leqq \varepsilon$ for any $\mu(E)<\infty$, and $E \uparrow_{\mu(\mathbb{B})<\infty} \Omega$ there exists $y_{0}=\bigcup_{\mu(\mathbb{E})<\infty} y_{E} \in L_{\Phi}$ with $m_{\Phi}\left(y_{0}\right) \leqq \varepsilon$ which is defined for all on $\Omega$, and so, $y_{0} \in L_{w}$ and $m_{w}\left(k y_{0}\right)<\gamma$ by the same reason stated above.

Thus, we have for all positive real numbers $\xi \geqq 0$ $\Psi(k \xi, \omega) \leqq K \Phi(\xi, \omega)+\Psi\left(k y_{0}(\omega), \omega\right)$ for a.e. on $\Omega$

by 5). $\Psi\left(k y_{0}(\omega), \omega\right)$ is no other than $c(\omega)$ in $(*)$.

Corollary 1. $L_{\Phi}$ is equivalent to $L_{T}$ if and only if there exist $k_{1}, k_{2}, K_{1}, K_{2}>0$ and $c \in L_{1}$ such that

$$
\left|K_{1} \Phi\left(k_{1} \xi, \omega\right)-K_{2} \Phi\left(k_{2} \xi, \omega\right)\right| \leqq c(\omega)
$$

for all $\xi \geqq 0$ and a.e. on $\Omega$.

Corollary 2. (1) Let $L_{M(\xi)}(\Omega)$ be an Orlicz space defined on $\Omega$ by a function $M(\xi)(\xi \geqq 0)$.

$L_{\emptyset} \subseteq L_{M}$ if and only if there exist $k, K>0$ and $c(\omega) \in L_{1}(\Omega)$ such that

$$
\Phi(k \xi, \omega) \leqq K M(\xi)+c(\omega)
$$

for all $\xi \geqq 0$ and a.e. on $\Omega$.

(2) Let $L_{p(\omega)}(\Omega)$ be a modular function space which is of unique spectra $^{6)}$ defined on $\Omega$ by a measurable function $1 \leqq p(\omega) \leqq \infty(\omega \in \Omega)$.

$L_{\oplus} \subseteq L_{p(\omega)}$ if and only if there exist $k, K>0$ and $c \in L_{1}$ such that $\Phi(k \xi, \omega) \leqq K \xi^{p(\omega)}+c(\omega)$

for all $\xi \geqq 0$ and a.e. on $\Omega$.

Next, we consider the case that $\mu$ is atomic.

If $\mu$ is atomic, we can assume $\mu(\omega)=1$ for all $\omega \in \Omega$ without loss of generality. And $m_{\Phi}(x)=\sum_{\omega \in \Omega} \Phi(|x(\omega)|, \omega)$ for all $x \in L_{\varphi}$.

Theorem 2. $L_{\mathscr{\Phi}} \subseteq L_{q}$ if and only if there exist $k, K>0, c(\omega) \geqq 0$ $(\omega \in \Omega)$ with $\sum_{\omega \in \Omega} c(\omega)<\infty$ and $\xi_{\omega}(\omega \in \Omega)$ which is a system of numbers satisfying, for any $x \in L_{\phi}$ with $m_{\Phi}(x)<\infty,|x(\omega)| \leqq \xi_{\omega}$ except on some finite subset of $\Omega$, such that

$$
\Psi(k \xi, \omega) \leqq K \Phi(\xi, \omega)+c(\omega)
$$

for all $\xi \leqq \xi_{\omega}$ and $\omega \in \Omega$.

Proof. If $(* *)$ is valid, for any $x \in L_{\varphi}$, since there exists $\alpha>0$

6) These spaces were defined and discussed precisely in $H$. Nakano $[3, \S 89]$, that is,

with the modular

$$
L_{p(\omega)}(\Omega)=\left\{x: \int_{\Omega}|\omega x(\omega)|^{p(\omega)} d \mu<\infty \text { for some } \alpha=\alpha_{x}>0\right\}
$$

$$
m(x)=\int_{\Omega} \frac{1}{p(\omega)}|x(\omega)|^{p(\omega)} d \mu
$$

for a measurable function $1 \leqq p(\omega) \leqq \infty$ on $\Omega$. 
with $m_{\Phi}(\alpha x)<\infty$, we can find a finite subset $F \subset \Omega$ such that $\alpha|x(\omega)|$ $\leqq \xi_{\omega}$ for all $\omega \bar{\epsilon} F$ and

for all $\omega \bar{\epsilon} F$.

$$
\Psi(k \alpha|x(\omega)|, \omega) \leqq K \Phi(\alpha|x(\omega)|, \omega)+c(\omega)
$$

Furthermore, there exists $k^{\prime}$ with $0<k^{\prime} \leqq \alpha k$ such that $\Psi\left(k^{\prime}|x(\omega)|, \omega\right)<\infty$ for all $\omega \in F$, because $0 \leqq|x(\omega)|<\infty$ and 7) on $\Psi$.

Thus, we have

$$
\begin{aligned}
\sum_{\omega \in \Omega} \Psi & \left(k^{\prime}|x(\omega)|, \omega\right) \\
& \leqq \sum_{\alpha|x(\omega)| \leq \xi_{\omega}} K \Phi(\alpha|x(\omega)|, \omega)+\sum_{\alpha|x(\omega)| \leq \xi_{\omega}} c(\omega)+\sum_{\alpha|x(\omega)|>\xi_{\omega}} \Psi\left(k^{\prime}|x(\omega)|, \omega\right) \\
& \leqq K \sum_{\omega \in \Omega} \Phi(\alpha|x(\omega)|, \omega)+\sum_{\omega \in \Omega} c(\omega)+\sum_{\omega \in H^{\prime}} \Psi\left(k^{\prime}|x(\omega)|, \omega\right)<\infty .
\end{aligned}
$$

Namely $x \in L_{\emptyset}$.

Conversely, if $L_{\Phi} \subseteq L_{\psi}$, then $(* *)$ is proved also by the analogous way to the case which $\mu$ is non-atomic as the following.

Let $0 \leqq \alpha_{\nu}(\nu=1,2, \cdots)$ be the system of all the positive rational numbers and let $\varepsilon^{\prime}$ and $\varepsilon$ in (I) be $2 \varepsilon^{\prime}<\varepsilon$. We put, for all $\omega \in \Omega$,

$$
\alpha_{\omega}=\sup \left\{\alpha ; \alpha>0, \Psi(k \alpha, \omega)>K \Phi(\alpha, \omega), \Phi(\alpha, \omega)<\varepsilon^{\prime}\right\}
$$

and

$$
x_{0}(\omega)=\bigcup_{\omega^{\prime} \in \Omega} \alpha_{\omega^{\prime}} \chi_{\omega^{\prime}}(\omega)=\bigcup_{H^{\prime} \subset \Omega} \bigcup_{\omega^{\prime} \in H^{\prime}} \alpha_{\omega^{\prime}} \chi_{\omega^{\prime}}(\omega)
$$

where every $\chi_{\omega^{\prime}}$ is the characteristic function of $\left\{\omega^{\prime}\right\}$ and $F$ is a finite subset of $\Omega$, respectively.

Then, because, for any two elements $\omega_{1}, \omega_{2} \in \Omega$,

and

$$
m_{\Phi}\left(\alpha_{\omega_{1}} \chi_{\omega_{1}} \bigcup \alpha_{\omega_{2}} \chi_{\omega_{2}}\right)=\Phi\left(\alpha_{\omega_{1}}, \omega_{1}\right)+\Phi\left(\alpha_{\omega_{2}}, \omega_{2}\right)<2 \varepsilon^{\prime}<\varepsilon
$$

$$
m_{\Psi}\left(k \alpha_{\omega_{1}} \chi_{\omega_{1}} \cup \alpha_{\omega_{2}} \chi_{\omega_{2}}\right)>K m_{\Phi}\left(\alpha_{\omega_{1}} \chi_{\omega_{1}} \cup \alpha_{\omega_{2}} \chi_{\omega_{2}}\right)
$$

we have $m_{\emptyset}\left(\bigcup_{\omega \in F^{*}} \alpha_{\omega} \chi_{\omega}\right)<\varepsilon^{\prime}$ by $(\mathrm{I})$.

Since $\bigcup_{\omega \in \mathcal{F}^{p}} \alpha_{\omega} \chi_{\omega} \uparrow_{F^{\prime} \subset \Omega} x_{0}$ and $m_{\Phi}$ is monotone complete, we have $x_{0} \in L_{\Phi}$ and $m_{\emptyset}\left(x_{0}\right) \leqq \varepsilon^{\prime}$. Namely $x_{0} \in L_{\Psi}$ by the hypothesis and $m_{\Psi}\left(k x_{0}\right) \leqq \gamma$ by (a) in $(I)$.

On the other hand, putting

$$
\xi_{\omega}=\sup _{\Phi(\xi, \omega) \leq \varepsilon} \xi
$$

then for any $x \in L_{\Phi}$ with $m_{\Phi}(x)=\sum_{\omega \in \Omega} \Phi(|x(\omega)|, \omega)<\infty$ we can find a finite subset $F_{x} \in \Omega$ such that $\Phi(|x(\omega)|, \omega) \leqq \varepsilon$ for all $\omega \bar{\epsilon} F_{x}$. Namely $|x(\omega)|$ $\leqq \xi_{\omega}$ for all $\omega \bar{\epsilon} F_{x}$.

Thus, we have, for all $\omega \in \Omega$

$$
\Phi(k \xi, \omega) \leqq \begin{cases}\Psi\left(k x_{0}(\omega), \omega\right) & \text { for all } 0 \leqq \xi<\alpha_{\omega} \\ K \Phi(\xi, \omega) & \text { for all } \alpha_{\omega} \leqq \xi \leqq \xi_{\omega}\end{cases}
$$

by (b) in (I). Therefore (**) is proved by putting

$$
c(\omega)=\Psi\left(k x_{0}(\omega), \omega\right)
$$

Similar conditions to the corollaries to Theorem 1 on the case that 
$\mu$ is non-atomic hold also in this case.

Finally, the author wishes to express his hearty gratitude to Professor Hidegorô Nakano for his guidance and frequent encouragement.

\section{References}

[1] I. Amemiya: A generalization of the theorem of Orlicz and Birmbaum, Jour. Fac. Sci. Hokkaidô Univ., ser. 1, 12, no. 2 (1956).

[2] H. Nakano: Modulared Semi-ordered Linear Spaces, Tokyo (1950).

[3] —-: Topology and Linear Topological Spaces, Tokyo (1951).

[4] - : Measure Theory (in Jap.), 3, Tokyo (1950).

[5] W. Orlicz and Z. Birnbaum: Über die Verallgemeinerung des Begriffes der zueinander konjugierten Potenzen, Studia Math., 3 (1931).

[6] T. Shimogaki: Note on Orlicz-Birnbaum-Amemiya's theorem, Proc. Japan Acad., 33, no. 6 (1957). 